\title{
Robotic mitral valve repair in a patient with cardiac dextroversion
}

Alexandra Bourdillon, BS, ${ }^{a}$ Robert Elder, MD, ${ }^{\mathrm{b}, \mathrm{c}}$ Michael Lalonde, MHA PA-C, ${ }^{\mathrm{d}}$ Jeremy Steele, MD, Peter J. Gruber, MD, PhD, ${ }^{\mathrm{d}}$ and Arnar Geirsson, MD, ${ }^{\mathrm{d}}$ New Haven, Conn

\footnotetext{
From the ${ }^{\mathrm{a} Y a l e}$ School of Medicine, New Haven, Conn; ${ }^{\mathrm{b}}$ Section of Pediatric Cardiology, Department of Pediatrics, ${ }^{\mathrm{c}}$ Section of Cardiovascular Medicine, Department of Internal Medicine, and ${ }^{\mathrm{d}}$ Division of Cardiac Surgery, Department of Surgery, Yale School of Medicine, New Haven, Conn.

Disclosures: Dr Geirsson reported a financial relationship with Edwards Lifesciences and Medtronic. All other authors reported no conflicts of interest.

The Journal policy requires editors and reviewers to disclose conflicts of interest and to decline handling or reviewing manuscripts for which they may have a conflict of interest. The editors and reviewers of this article have no conflicts of interest.

Received for publication May 11, 2021; accepted for publication Oct 15, 2021; available ahead of print Oct 23, 2021.

Address for reprints: Arnar Geirsson, MD, 330 Cedar St, Boardman 204, New Haven, CT 06519 (E-mail: arnar. geirsson@yale.edu).

JTCVS Techniques 2022;11:12-6

2666-2507

Copyright (c) 2021 The Author(s). Published by Elsevier Inc. on behalf of The American Association for Thoracic Surgery. This is an open access article under the CC BY-NC-ND license (http://creativecommons.org/licenses/bync-nd/4.0/).

https://doi.org/10.1016/j.xjtc.2021.10.031
}

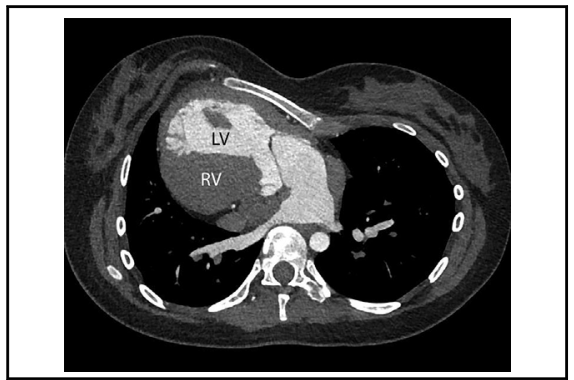

Cardiac dextroversion with morphological left ventricle ( $L V$ ) positioned anterior to right ventricle (RV).

CENTRAL MESSAGE

Mitral regurgitation and left ventricular outflow tract obstruction can be effectively approached and treated with complex robotic mitral valve repair in a patient with cardiac dextroversion.

See Commentaries on pages 17 and 19.
A 25-year-old woman with dextroversion was found to have moderate mitral regurgitation (MR) complicated by systolic anterior motion (SAM) of the anterior leaflet, causing obstruction of the aortic valve and left ventricular outflow tract (LVOT). The patient presented with increasing symptoms of intermittent nonexertional chest pressure and dyspnea on exertion. A preoperative transthoracic echocardiogram revealed worsening $\mathrm{MR}$ and LVOT obstruction with a peak resting gradient of $37 \mathrm{~mm} \mathrm{Hg}$ and $70 \mathrm{~mm} \mathrm{Hg}$ on provocative testing. Further imaging with computed tomography and magnetic resonance imaging was obtained for surgical planning employing the da Vinci $\mathrm{Xi}$ surgical robot (Intuitive Surgical, Inc, Sunnyvale, Calif). The heart was positioned in the right chest, with the apex pointing towards the right. The left ventricle was anterior to the right ventricle, with the mitral valve positioned anterior to the aortic valve and LVOT (Figure E1, $A$ ). It was noted that the posteriorly leaflet was quite long and that the acute angle from the anterior leaflet of the mitral valve to the aortic root was causing the LVOT obstruction (Figure E1, B). Intraoperative transesophageal echocardiogram showed SAM, moderate MR, anterior mitral leaflet height of $4.0 \mathrm{~cm}$, posterior mitral leaflet height of
$2.50 \mathrm{~cm}$, LVOT of $1.1 \mathrm{~cm}$, and C-sept distance of $2.39 \mathrm{~cm}$ (Figure E2, A).

The patient was positioned supine with gel pad under the left side to rotate the chest slightly toward the right. A small access incision was made in the left fourth intercostal space and robotic ports placed in second, fourth, and sixth intercostal space (Figure 1, A). Femoral cannulation was used for cardiopulmonary bypass and IntraClude (Edwards Lifesciences) intra-aortic occlusion device for cardioplegia delivery. The robot was connected in place, the left lung was deflated, and the chest was explored. Exposure through the pericardium was carefully achieved without injury to the left phrenic nerve, which was running quite anteriorly on the pericardium. Cardiopulmonary bypass was initiated, and cardioplegia was administered after confirming a robust seal of the inflated endoballoon. The left atrium was entered just above the left superior pulmonary veins and posterior to the coronary sinus, which contained the persistent left superior vena cava. A retractor placed for exposure to the mitral valve, which 


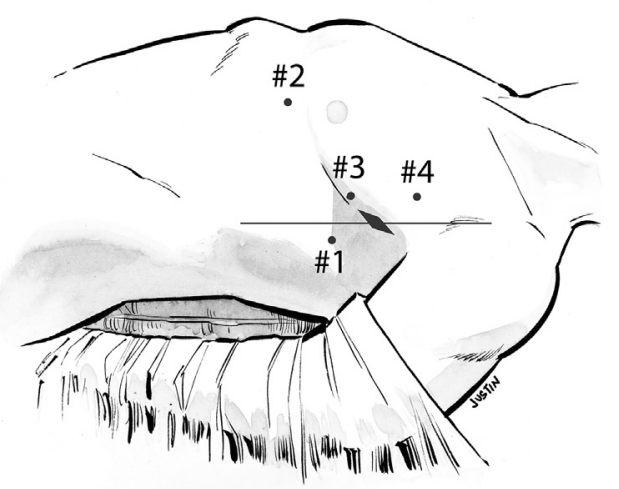

A

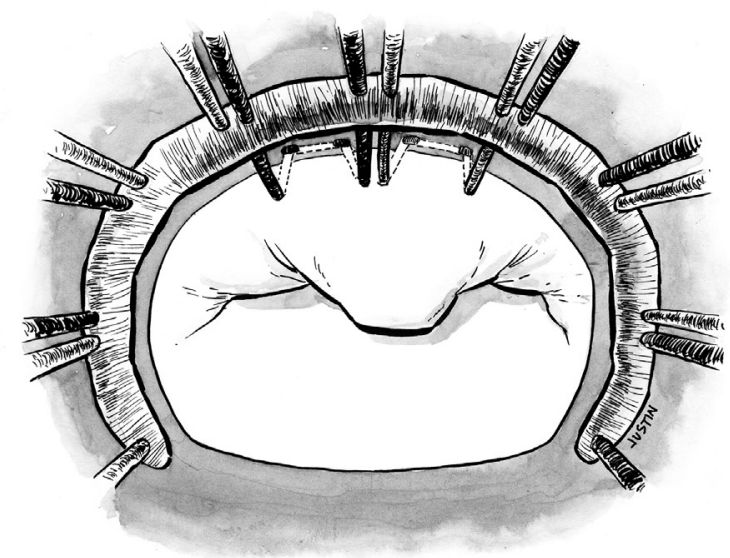

B

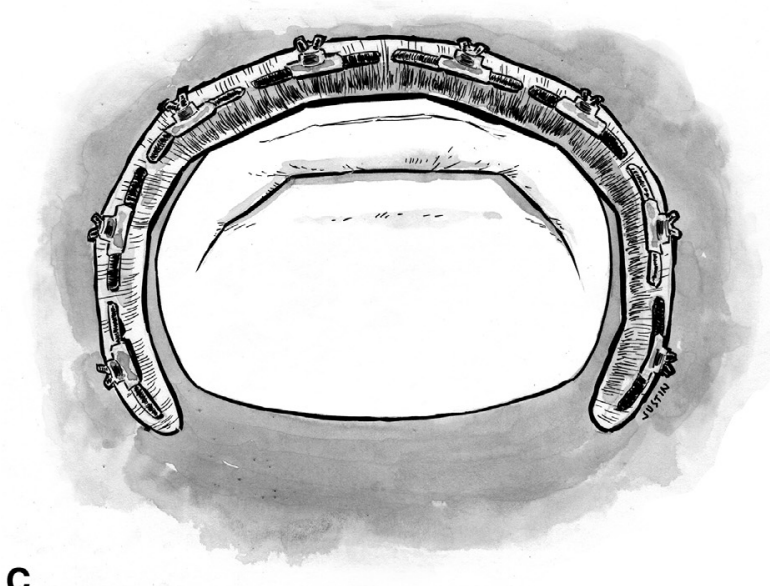

FIGURE 1. Illustrations depicting operative technique including (A) port placement for left-sided approach from proximal to distal: right arm port second intercostal space (ICS) (arm \#4), working and camera port fourth ICS (arm \#3), left atrial retractor port fifth ICS (arm \#2), and left arm port sixth ICS (arm \#1). B, View from surgeon's perspective of the annular advancement repair technique of the morphologically posterior leaflet in its anterior location. C, View from surgeon's perspective after repair.

was uniquely oriented in that the morphologically posterior leaflet faced anteriorly (Figure 2, A). No leaflet prolapse was observed (Figure 2, B).
The valve was repaired by decreasing the height of the posterior leaflet using annular advancement technique also known as imbrication technique. ${ }^{1}$ This decreases the height of the leaflet without resection of leaflet tissue and is a useful, nonresectional technique that decreases the risk of SAM after mitral valve repair. Each arm of horizontal mattress annuloplasty sutures is brought through the annulus and advanced 3 to $4 \mathrm{~mm}$ into the leaflet itself in the area of excessive length (Figures $1, B$, and 2,C). This effectively decrease the height of the leaflet (Figure 1,C). A 32-mm Simulus Semi-Rigid Annuloplasty Band (Medtronic, Minneapolis, Minn) was selected. This particular band is semirigid and was used to further elevate the posterior leaflet away from the anterior leaflet and the LVOT. An additional suture was placed to close a small cleft between $\mathrm{P} 1$ and $\mathrm{P} 2$. Intraoperative assessment was satisfactory after repair (Figure 2, D). The heart was deaired, the endoballoon was deflated, and the patient was weaned off bypass without complication. Good function of the mitral valve with trace to mild MR, without SAM or LVOT obstruction, was confirmed under echo (Figure E2, B). Video 1 shows the conduct of the operation and imaging studies. Written consent for the patient's authorization of disclosure was obtained in compliance with the Health Insurance Portability and Accountability Act of 1996 and Yale institutional review board protocols.

\section{DISCUSSION}

Dextroversion is defined by right-sided cardiac positioning with a rightward cardiac apex in the setting of situs solitus. In contrast to situs inversus, in which the visceroatrial orientation mirrors the normal organization, in dextroversion there is normal positioning of tracheobronchial tree and abdominal viscera. ${ }^{2}$ It is thought that this malposition occurs because of a failure of the cardiac apex to redirect from right-facing to left-facing during the ninth week of embryologic development. ${ }^{3}$ In the literature, it has been reported that approximately $40 \%$ of patients with dextrocardia belong to each of these 2 orientations, whereas just $20 \%$ of patients are classified by a third orientation situs ambiguus, which designates anatomical orientations that possess a mixture of features similar to both situs solitus and situs inversus. ${ }^{2}$ Dextrocardia is rare and predisposes to congenital heart disease, and it may be more complicated in patients with situs solitus compared with situs inversus. ${ }^{2,4}$

Robotic surgery has been employed for mitral valve surgery since the 1990s and has been shown to rival or exceed outcomes in open surgeries, although its popularity has been stunted by associated costs and labor-intensive training. ${ }^{5}$ In dextrocardia, the pulmonary trunk may lie posterior and left of the aorta. This and other shifts in anatomical relationships require significant surgical finesse to employ traditional robotic surgery techniques for patients with this condition. Although the literature appears to 


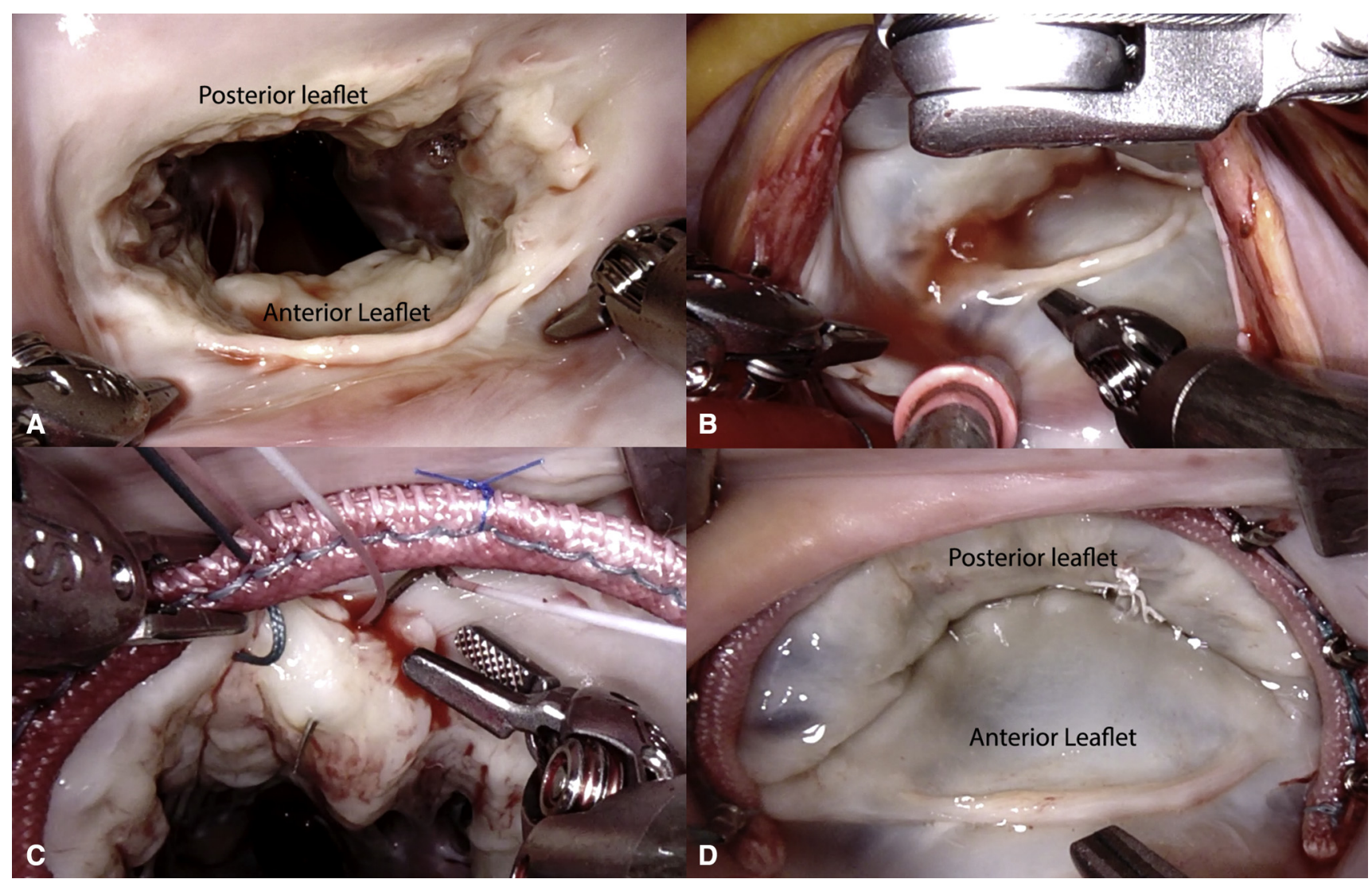

FIGURE 2. Intraoperative picture showing (A) morphologic anterior mitral valve leaflet positioned inferiorly; (B) saline testing before mitral valve repair with coaptation line in center of the valve due to excessive length of morphological posterior leaflet; $(C)$ annular advancement technique used to effectively shorten the height of the morphological posterior leaflet; and (D) saline testing after mitral valve repair with semirigid annuloplasty band restoring the normal one-third posterior to two-thirds anterior height ratio.

contain an increasing number of cases of endoscopic surgical repairs in patients with dextrocardia and situs inversus, ${ }^{6}$ no publication to date has reported a similar robotic

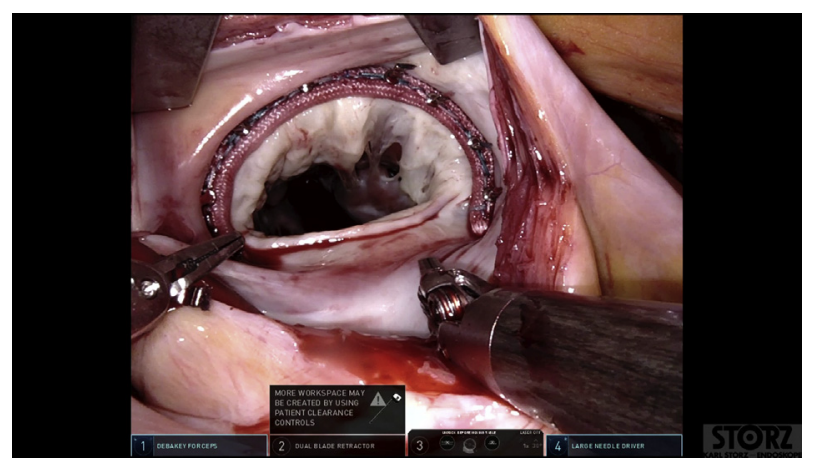

VIDEO 1. Video demonstration of the operative technique employed for mitral valve repair in a case of dextroversion. Video available at: https:// www.jtcvs.org/article/S2666-2507(21)00722-7/fulltext. approach to mitral valve repair in a patient with dextroversion. This report offers a novel and safe approach to treat MR in patients with these complex anatomical relationships.

\section{References}

1. Hashim PW, Assi R, Hashim SW. The imbrication technique: an alternative to the sliding leaflet technique. Ann Thorac Surg. 2014;98:1124-6.

2. Tripathi S, Ajit Kumar VK. Comparison of morphologic findings in patients with dextrocardia with situs solitus vs situs inversus: a retrospective study. Pediatr Cardiol. 2019;40:302-9.

3. De la Cruz MV, Anselmi G, Munos-Castellanos L, Nadal-Ginard B, Munoz-Armas S. Systematization and embryological and anatomical study of mirror-image dextrocardias, dextroversions, and laevoversions. Br Heart J. 1971;33:841-53.

4. Offen S, Jackson D, Canniffe C, Choudhary P, Celermajer DS. Dextrocardia in adults with congenital heart disease. Heart Lung Circ. 2016;25:352-7.

5. Sultan I, Trivedi DP, Machiraju VR. Surgical approaches to the mitral valve: variable paths to the same destination. Indian J Thorac Cardiovasc Surg. 2018;34 $105-12$.

6. Iino K, Watanabe G, Ishikawa N, Tomita S. Total endoscopic robotic atrial septal defect repair in a patient with dextrocardiac and situs inversus totalis. Interact Cardiovasc Thorac Surg. 2012;14:476-7. 


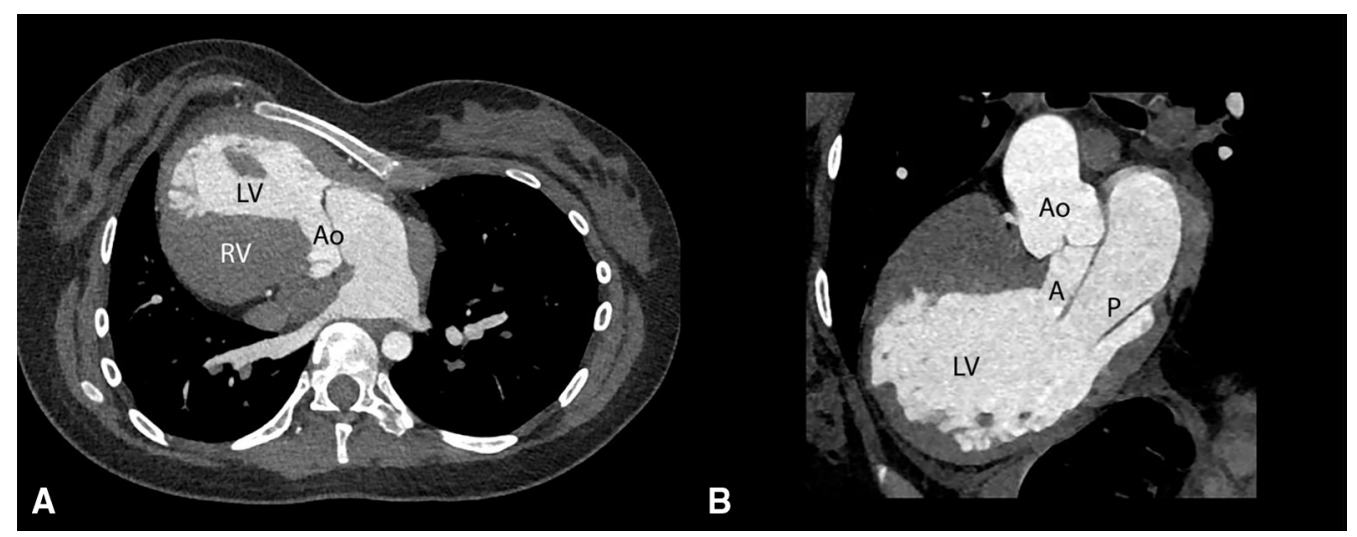

FIGURE E1. Computed tomography angiography demonstrating (A) cardiac dextroversion with heart rotated into right chest where the left ventricle ( $L V$ ) is situated anterior to both right ventricle $(R V)$ and the aortic root $(A o)$; and $(\mathrm{B})$ an acute aortomitral angle is noted with near-equal length of anterior and posterior mitral leaflets. 


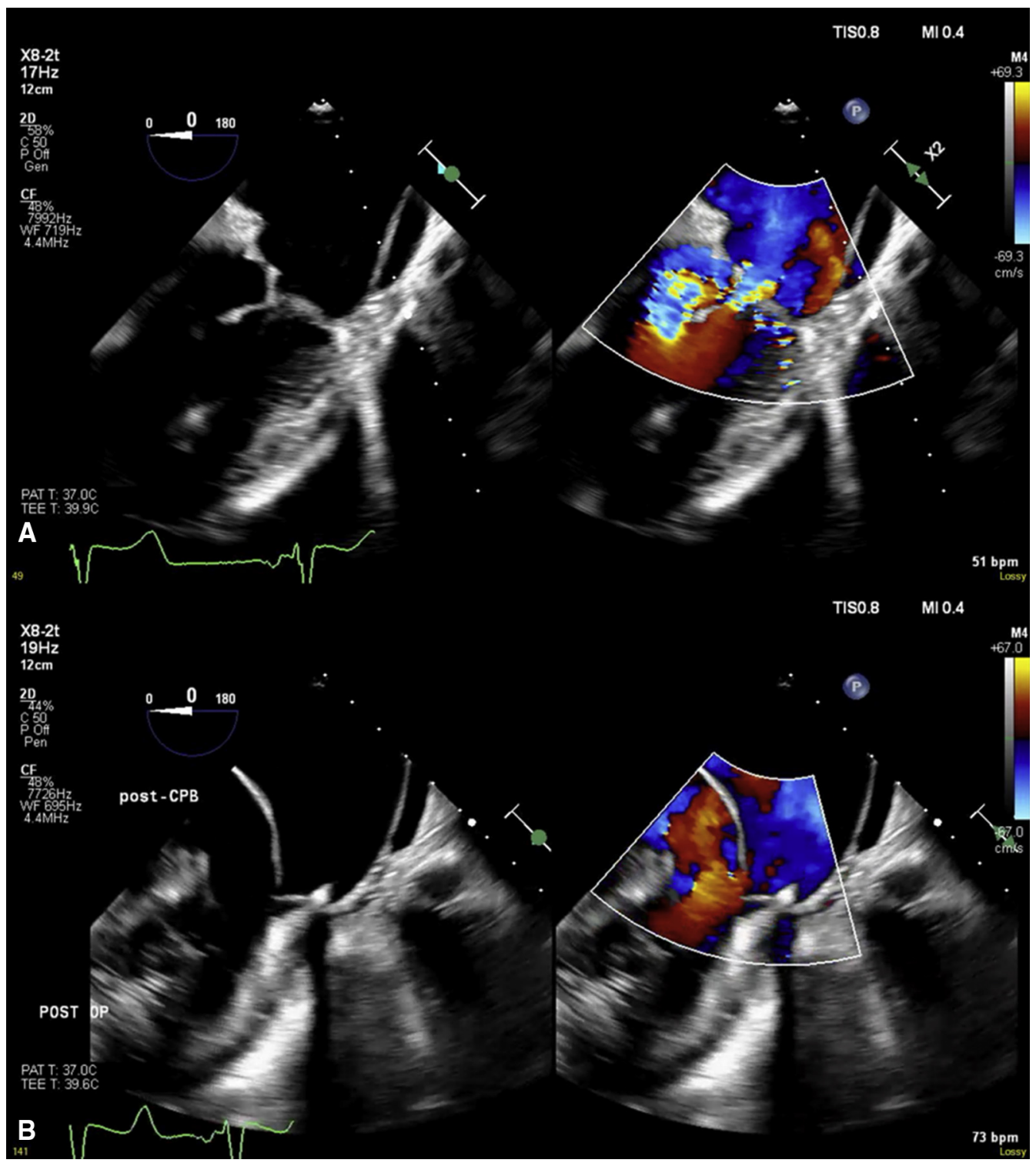

FIGURE E2. Intraoperative transthoracic echocardiography demonstrating (A) prerepair systolic anterior motion (SAM) of the anterior leaflet with moderate mitral regurgitation; and (B) postrepair with no SAM or significant mitral regurgitation. 